ISSN 1991-8631

Original paper

http://indexmedicus.afro.who.int

\title{
Comportement de quelques lignées de riz NERICA en culture de bas-fond dans la région de Kinshasa, République Démocratique du Congo (RDC)
}

\author{
B.M. BANGATA ${ }^{1 *}$, K.N. NGBOLUA ${ }^{2}$, E. EKUTSU ${ }^{2}$ et A. KALONJI-MBUYI ${ }^{1}$ \\ ${ }^{I}$ Département de Phytotechnie, Faculté des Sciences Agronomiques, Université de Kinshasa, \\ BP.117 Kinshasa XI, République Démocratique du Congo. \\ ${ }^{2}$ Département de Biologie, Faculté des Sciences, Université de Kinshasa, BP. 190 Kinshasa XI, \\ République Démocratique du Congo. \\ *Auteur correspondant, E-mail : bithanyimbunzu@yahoo.fr; Tél. : +243813630475
}

\section{RESUME}

Une étude visant l'accroissement de la production locale de variétés améliorées du riz de bas-fond a été menée à Kinshasa entre les mois d'Octobre 2009 et Mars 2010. Cette étude avait pour but d'évaluer la performance de quatre lignées de riz de variété améliorée NERICA de bas-fond en vue d'identifier la meilleure lignée à vulgariser en République Démocratique du Congo (RDC). Les résultats de l'essai conduit suivant un dispositif en blocs complets randomisés avec quatre répétitions ont montré que toutes les quatre lignées testées sont bonnes, présentant un poids de 1000 grains élevé. Cependant, du point de vue numérique, la lignée NL-16 présente le meilleur poids $(30,43 \pm 1,63$ gr). A cet effet, toutes ces lignées peuvent être retenues et recommandées comme variétés de culture en mettant un accent particulier sur la lignée NL-16.

(C) 2013 International Formulae Group. All rights reserved.

Mots clés: Productivité, pyriculariose, RDC.

\section{INTRODUCTION}

A l'heure actuelle, les populations des pays en développement et en particulier ceux d'Afrique Subsaharienne connaissent une crise alimentaire sans précédent. Cette crise a révélé les limites des politiques agricoles mises en œuvre jusqu'à ce jour par les pouvoirs publics en vue d'assurer la sécurité alimentaire et dont l'une des composantes principales est l'importation du riz.

Comme solution alternative à cette crise alimentaire, plusieurs pays essaient de trouver des solutions durables par la mise en place d'une politique d'autosuffisance alimentaire grâce à l'auto-approvisionnement en riz (Yovo, 2008).

La RDC dispose de potentialités énormes en ressources naturelles. Cependant, malgré cet énorme potentiel, le pays est classé parmi les plus pauvres du continent. En effet, près de $75 \%$ de la population congolaise souffre de malnutrition (Tollens et Biloso, 2006). Il est donc indispensable de définir des nouvelles stratégies plus appropriées en vue d'accroître la disponibilité de cette denrée localement pour le bien être de la population démunie comme celle de l'Afrique. C'est dans ce cadre que le Centre de Riz pour l'Afrique ADRAO, a au cours de ces dernières années, 
mis un accent particulier sur la recherche et le développement participatif afin de réadapter les systèmes de production de riz aux nouvelles innovations technologiques afin de faire face aux contraintes démographiques (Sido et al., 2006).

Le Nouveau Riz pour l'Afrique ou "New Rice for Africa" (NERICA) est le produit d'une hybridation interspécifique entre les espèces de riz africain et asiatique s'adaptant aux contraintes locales du riz africain et ayant le rendement potentiel élevé du riz asiatique (augmentation de 50\% sans engrais et de plus de $200 \%$ avec engrais) (Koudjega et al., 2006; Kone et al., 2008; Cissoko et al., 2011). L'intérêt du riz NERICA est évident.

En effet, c'est un riz qui produirait bien avec un minimum d'intrants dans divers ensembles agroécologiques africains et qui répondrait encore mieux lorsque les paysans sont en mesure d'appliquer des intrants supplémentaires et la teneur en protéines est plus élevée (2\%). Il existe aujourd'hui de NERICA pluvial et de NERICA de bas-fond (Tshibambe, 2006). Malgré cet intérêt, la production locale reste toujours faible, en raison principalement des attaques des maladies, des ravageurs et autres oiseaux qui constituent un facteur limitant de la production en RDC (Nseye et al., 2009). Parmi les affections les plus importantes de la culture du riz, il y a la pyriculariose, maladie causée par le champignon Pyricularia oryzae.

Cette maladie cryptogamique, la plus redoutée en zones rizicoles de la RDC (Nseye et al., 2009), peut se manifester sur toutes les parties aériennes du riz, les gaines, les limbes, les rachis de panicule et parfois les glumelles. Les conditions climatiques et édaphiques ont une grande influence sur l'apparition et l'évolution de la maladie. Lorsque l'infection apparaît précocement, les plants demeurent rabougris (Kasongo et al., 2003).

Les dégâts en pépinière ou au tallage peuvent entrainer la destruction complète du plant, d'où l'intérêt de déterminer le comportement de matériel en introduction visà-vis des attaques possible de la pyriculariose (Angladette, 1966; Bouet et al., 2006). Dans ce travail, nous avons évalué le niveau de résistance à la pyriculariose et les caractéristiques agronomiques de quatre lignées de riz NERICA de bas-fond afin d'identifier la lignée présentant la meilleure performance en vue de la vulgarisation de son exploitation.

\section{MATERIEL ET METHODES}

\section{Site expérimental}

L'expérimentation a été conduite à N'djili brasserie situé à environ 30 kilomètres du centre ville de Kinshasa ( $4^{\circ} 29^{\prime}$ de latitude Sud et $15^{\circ} 23^{\prime}$ de longitude Est, 471,31 m d'altitude) en RDC. Les précédents culturaux étaient constitués d'une jachère dominée par Cynodon dactylon, Panicum repens et Cyperus rotundus. Le sol présente une texture argileuse dans le bas fond marécageux. L'essai a été conduit du 10 octobre 2009 au 05 mars 2010 (saison culturale A/2010). La température pendant la période de l'essai a variée entre 25,2 et $26,9^{\circ} \mathrm{C}$.

\section{Matériel végétal}

Quatre lignées de riz de la variété NERICA de bas-fond à cycle moyen (N-L16, N-L17, N-L19 et N-L56) provenant de l'ADRAO en Côte d'Ivoire ont été utilisées dans cette étude. La variété locale de riz SIPI a été utilisée comme témoin. Toutes les semences ont été fournies par le Programme National Riz (PNR).

\section{Méthodes}

L'essai a été conduit suivant un dispositif en blocs complets randomisés avec 4 répétitions. Chaque bloc, représentant une répétition, comporte 5 parcelles correspondant aux lignés étudiées, soit un total de 20 parcelles. Les dimensions des parcelles ont été de $4 \mathrm{~m}$ de long et $3 \mathrm{~m}$ de large (soit $12 \mathrm{~m}^{2}$ ). Deux blocs consécutifs étaient séparés d'une allée de $1 \mathrm{~m}$, tandis que deux parcelles 
consécutives étaient distantes de 0,40 m. Le terrain a été labouré et les parcelles ont été tracées et piquetées avant le repiquage. Le pouvoir germinatif a été évalué selon Kabuyaya (2001).

Pour la pré-germination, $1 \mathrm{~kg}$ de semences de chacune des lignées ont été trempées dans l'eau pendant 12 heures. Ces semences sont ensuite retirées et égouttées pendant 48 heures. Un semis à la volée a été effectué avec des grains pré-germés, sur une plate-bande préalablement labourée de $14,5 \mathrm{~m}$ de long et de 1,20 m de large. Les variétés ont été séparées d'une allée mesurant $40 \mathrm{~cm}$ et toute la plate-bande a été couverte des pailles pendant 7 jours. Les plantules ont été repiquées au $27^{\text {ème }}$ jour après semis aux écartements de $20 \mathrm{~cm}$ entre les lignes et $20 \mathrm{~cm}$ dans la ligne (Halidou et al., 2006).

Le N-P-K 17-17-17 a été épandu à une dose de $200 \mathrm{~kg} / \mathrm{ha}$. Ceci était utilisé comme engrais de fond pour prévenir la carence. L'Urée $(46 \%)$ à la dose de $100 \mathrm{~kg} / \mathrm{ha}$ a été utilisée comme engrais de couverture. Ce dernier était fractionné en deux c'est-à-dire $50 \%$ de la quantité soit $1,2 \mathrm{~kg}$ appliqué au tallage et $50 \%$ appliqué à la montaison, conformément aux normes de l'IRRI (Narteh et al., 2006).

Les données ont été enregistrées à partir d'un échantillon de 10 plants choisis au hasard par parcelle expérimentale. Les caractéristiques agronomiques et les paramètres de rendement ont été évalués selon les critères et échelles établis par Kabuyaya (2001).

\section{Analyse statistique}

L'analyse de la variance à un facteur a été utilisée en vue de comparer les moyennes des variables étudiées au seuil de signification $\alpha=0,05$. Lorsque des différences sont observées, l'analyse est complétée par le test de la plus petite différence significative. Tous ces tests sont effectués à l'aide du logiciel $\mathrm{R}$ (version 2.9.0).

\section{RESULTATS \\ Qualité des semences utilisées}

Le résultat relatif au pouvoir germinatif des grains de quatre lignées de riz NERICA par rapport au témoin SIPI indique que toutes les lignées utilisées sont de bonne qualité car leur pouvoir germinatif est supérieur à $85 \%$ et répond aux normes de l'Institut International de Recherche sur le Riz (IRRI, 1980). En outre, l'analyse statistique révèle une différence significative entre les lignées $(\mathrm{LSD}=2,8)$. Le pourcentage de germination le plus élevé a été enregistré chez la lignée NERICA L-16 (98\%), suivi de NERICA L-56 $(96 \%)$ et le NL-19 (92\%). Le témoin SIPI (90\%) et le NL-17 (90\%) ont présenté un pourcentage inférieur aux autres lignées mises en compétition.

\section{Durée semi-floraison}

Les résultats relatifs au pouvoir germinatif des grains, la durée semisfloraison; la hauteur des plants; la longueur des panicules et le nombre de ramifications, tous en fonction des lignées/variétés sont présentés dans le Tableau 1.

Il ressort de notre étude que d'après l'échelle de cotation décrit par Kabuyaya (2001), nos matériels sont tous des variétés à cycle moyen car leur $50 \%$ jour à la floraison est compris entre 76 et 85 jours. Ce résultat est conforme aux travaux antérieurs (ADRAO, 2003) et permet de considérer les lignées de riz NERICA comme particulièrement intéressantes en termes de rendement et de gain de temps. En effet, il a été démontré que de telles particularités favorisent la répétition de la culture au cours de l'année et une bonne productivité par la bonne formation des grains (PNR, 1993).

\section{Hauteur des plants}

Les données sur la hauteur des plantes recueillies à la maturation (soit 118 jours après le repiquage) sont consignées dans le Tableau 1 et indiquent que les lignées NL-16, NL-17 et NL-56 présentent une hauteur 
moyenne (101 et $125 \mathrm{~cm}$ ). Tandis que NL-19 et SIPI présentent une hauteur courte (76 et $100 \mathrm{~cm}$ ) selon l'échelle de Kabuyaya (2001). L'analyse statistique révèle l'existence d'une différence significative entre les lignées au seuil de 5\%. (LSD = 11,4). Les valeurs observées sont d'une manière globale inférieure pour l'ensemble à celles fournies par les normes de l'Institut International de Recherche sur le Riz (IRRI, 1980).

\section{Longueur des panicules}

La longueur des panicules des lignées de riz NERICA par rapport au témoin SIPI a montré que la lignée NL-56 présente une longueur moyenne plus élevée $(26,1 \mathrm{~cm})$ suivi de NL-17 (25,5 cm) et NL-16 (25,4 $\mathrm{cm})$. Une faible longueur est observée chez la lignée NL-19 $(23,3 \mathrm{~cm})$. L'analyse statistique au seuil de probabilité 0,05 révèle une différence significative (LSD $0,05=1,8)$ entre les lignées utilisées.

\section{Nombre de ramifications}

Le nombre moyen de ramifications compté sur chaque lignée indique que les lignées NL-16 et N-17 présentent un nombre élevé de ramification que les autres. En outre, NL-19 a présenté moins de ramification (résultat repris dans le Tableau 1). L'analyse statistique au seuil de probabilité $\alpha=0,05$ indique qu'il existe une différence significative entre la lignée NL-19 $\left(\mathrm{LSD}_{0,05}=\right.$ $1,8)$ et les autres lignées. Cependant, aucune différence statistique ne se dégage entre les quatre autres lignées.

\section{Poids de 25 panicules}

Les mesures de poids effectuées sur un échantillon de 25 panicules pour chaque lignée sont reprises dans la Figure 1.

Il découle de cette figure une différence significative entre les paramètres observés
$\left(\mathrm{LSD}_{0,05}=20,5\right)$. L'analyse statistique a permis de regrouper le matériel en deux groupes: le premier groupe renferme les lignées NL-17, NL-16 et NL-56 dont le poids des panicules se situe entre 81,4 gr et 97,1 gr. Le second avec les lignées NL-19 et SIPI, présentant des faibles poids (67,3 gr- 74,8 gr). Ainsi donc, NL-17 semble avoir un poids élevé contrairement au témoin SIPI qui présente le faible poids.

\section{Pourcentage de stérilité}

La Figure 2 présente les niveaux de stérilité observée des lignées utilisées. Comme on peut l'observer sur la Figure 2, le niveau de stérilité le plus élevé est observé chez la lignée NL-56 tandis que la plus faible valeur est observée chez la lignée NL-19. En effet, Courtois et Taillebois (1990) ont étudié le comportement en androgénèse de lignées de riz porteuses d'une stérilité mâle d'origine génocytoplasmique et ont conclus que cette stérilité provoque généralement un avortement des microspores au stade uninucléé, et un avortement plus tardif des graines de pollen, entre le stade binucléé et trinucléé.

\section{Poids de 1000 grains (gr)}

Les poids de 1000 grains obtenus chez les lignées NERICA sont rapportés dans la Figure 3. Il ressort de cette figure que les poids de mille grains des lignées NL-16, NL19 et NL-56, variant de 29,85 à 30,43 gr, sont supérieurs au témoin SIPI sauf le NL-17 qui présente une masse de mille grains inférieur au témoin. En effet, ce résultat permet de considérer ce paramètre comme critère de choix de ces variétés pour leur diffusion. Le contrôle phytosanitaire effectué régulièrement tout au long de notre expérimentation n'a pas révélé la présence de la pyriculariose. Autrement dit, toutes ces variétés ont exprimé une résistance vis-à-vis de la pyriculariose. 


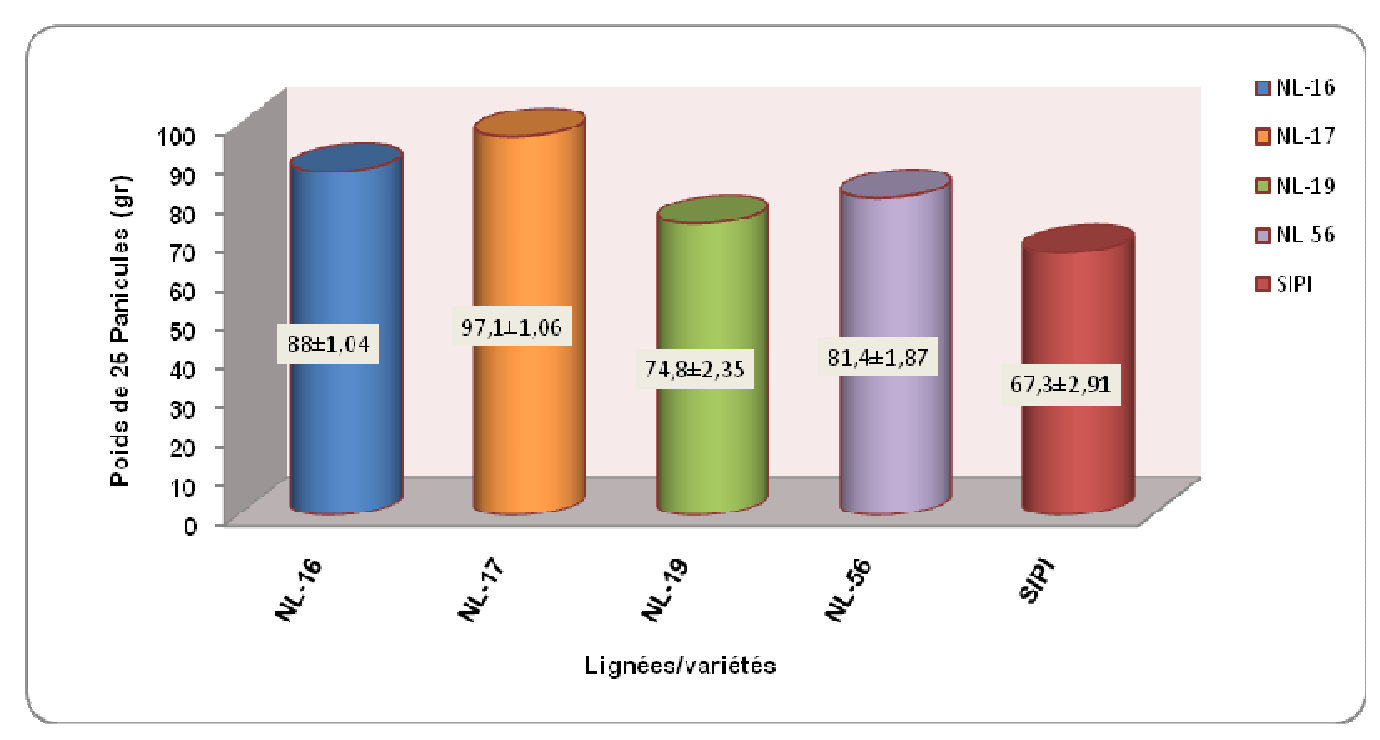

Figure 1: Le poids de 25 panicules en fonction des lignées/variétés.

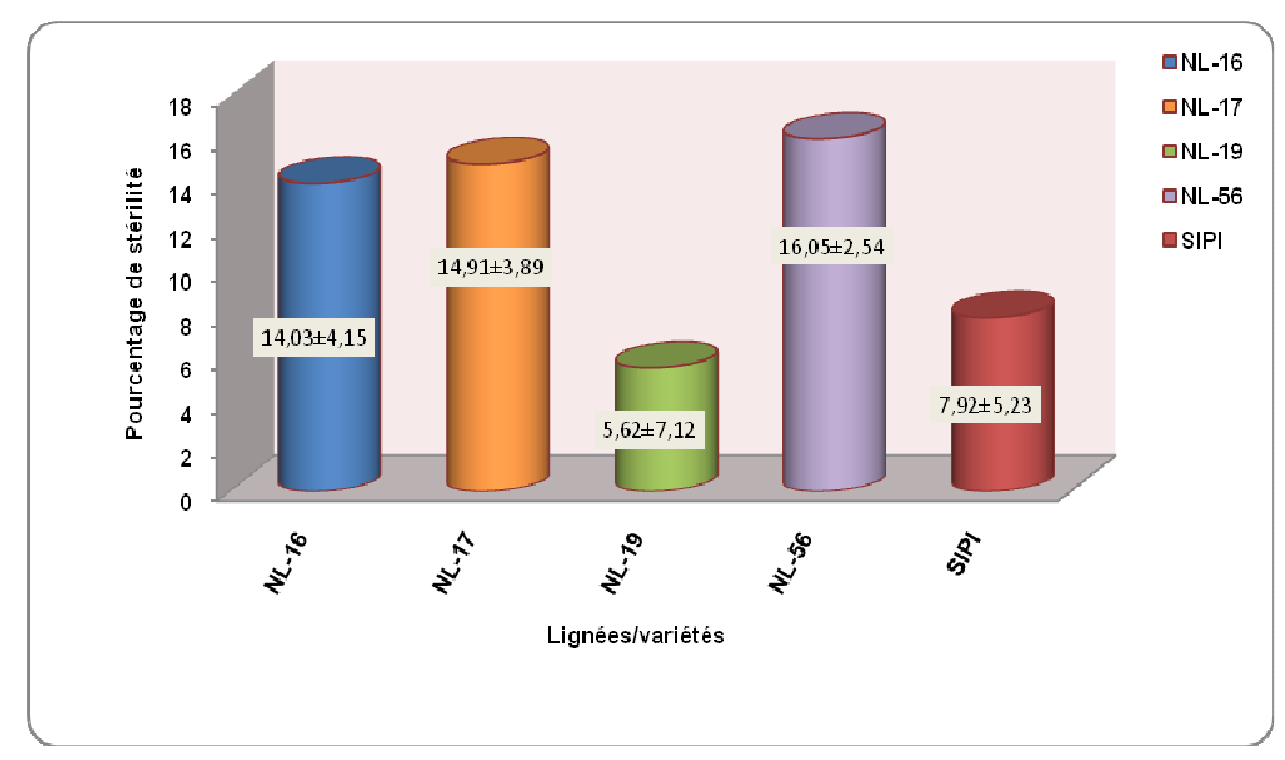

Figure 2: Le pourcentage de stérilité en fonction des lignées/variétés. 


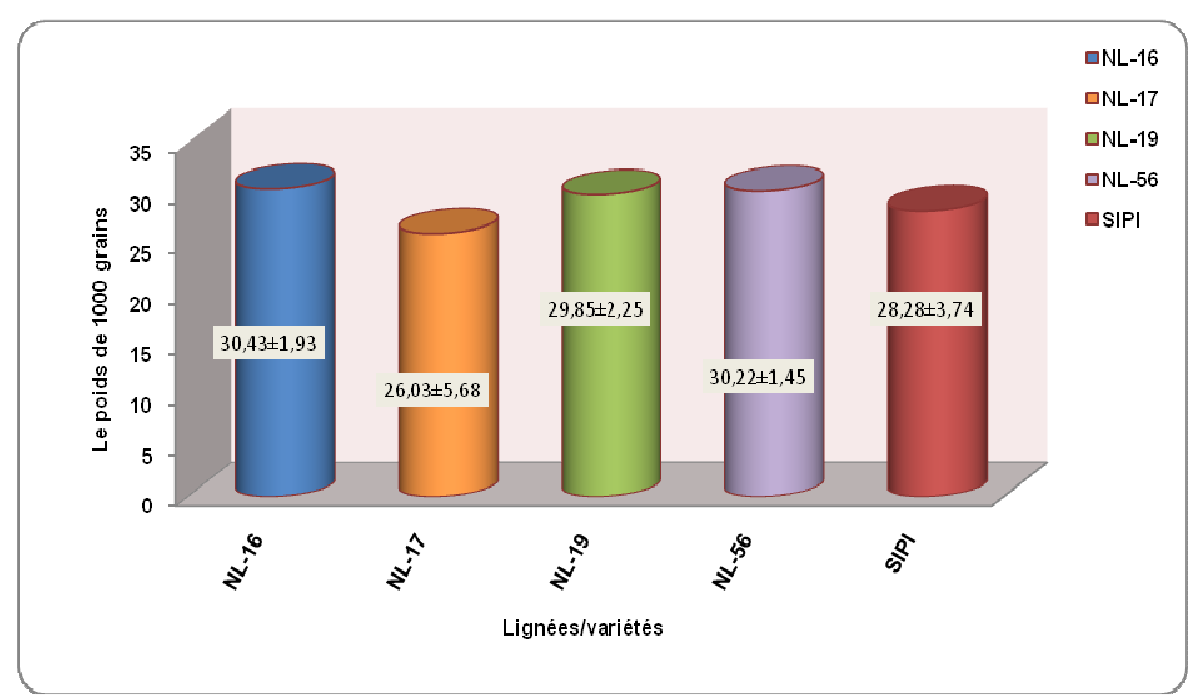

Figure 3: Le poids de 1000 grains en fonction des lignées/variétés.

Tableau 1: Le pourcentage de germination; la durée semis-floraison; la hauteur des plants; la longueur des panicules et le nombre de ramifications, tous en fonction des lignées/variétés.

\begin{tabular}{lccccc}
\hline $\begin{array}{l}\text { Lignées/ } \\
\text { variétés }\end{array}$ & $\begin{array}{c}\text { Pourcentage de } \\
\text { germination }\end{array}$ & $\begin{array}{c}\text { Durée semi- } \\
\text { floraison } \\
\text { (jours) }\end{array}$ & $\begin{array}{c}\text { Hauteur des } \\
\text { plants }(\mathbf{c m})\end{array}$ & $\begin{array}{c}\text { Longueur des } \\
\text { panicules }\end{array}$ & $\begin{array}{c}\text { Nombre de } \\
\text { ramifications }\end{array}$ \\
\hline SIPI & $90 \pm 10,8 \mathrm{a}$ & $81 \pm 12,15 \mathrm{a}$ & $97,67 \pm 8,3 \mathrm{~b}$ & $25,08 \pm 1,31 \mathrm{ab}$ & $9,5 \pm 1,21 \mathrm{a}$ \\
NL-16 & $98 \pm 8,82 \mathrm{~b}$ & $85 \pm 11,05 \mathrm{~b}$ & $101,83 \pm 7,43 \mathrm{ab}$ & $25,4 \pm 1,12 \mathrm{a}$ & $10,13 \pm 1,63 \mathrm{a}$ \\
NL-17 & $90 \pm 11,25 \mathrm{a}$ & $85 \pm 11,05 \mathrm{~b}$ & $106,61 \pm 6,55 \mathrm{ab}$ & $25,55 \pm 1,41 \mathrm{a}$ & $10,35 \pm 1,34 \mathrm{a}$ \\
NL-19 & $92 \pm 11,96 \mathrm{a}$ & $83 \pm 11,62 \mathrm{ab}$ & $98,07 \pm 7,15 \mathrm{~b}$ & $23,35 \pm 1,31 \mathrm{~b}$ & $7,7 \pm 1,13 \mathrm{ab}$ \\
NL-56 & $96 \pm 8,64 \mathrm{~b}$ & $85 \pm 11,05 \mathrm{~b}$ & $112,9 \pm 7,7 \mathrm{a}$ & $26,1 \pm 1,27 \mathrm{a}$ & $9,33 \pm 1,16 \mathrm{a}$ \\
\hline
\end{tabular}

Les chiffres dans les colonnes suivis de même lettre ne sont pas significativement différents selon le test de la Plus Petite Différence Significative (PPDS) à 5\% de probabilité.

\section{DISCUSSION}

En régions tropicales, la culture de riz est soumise à des contraintes biotiques et abiotiques drastiques conduisant à des menaces de leur érosion génétique (ADRAO, 2003).

Dans ce contexte, l'hybridation somatique est une approche prometteuse permettant de mettre au point des variétés capables de résister aux conditions des régions tropicales et d'augmenter la productivité. Le riz NERICA da bas-fond présente le double avantages qui fait défaut chez les deux parents (O. sativa et $O$. glaberrima). Les NERICA de bas-fond les plus prometteuses ont montré une faible sensibilité aux maladies et à l'attaque des insectes, ce qui est vital pour un biotope difficile comme le bas-fond.

Des résultats encourageants similaires concernant la résistance de ces lignées vis-àvis des parasites et visant la vulgarisation du riz NERICA dans d'autres régions d'Afrique ont été rapportés dans la littérature (Kone et al., 2008; Cissoko et al., 2011).

La RDC importe annuellement plus de 200000 tonnes de riz, en dépit de son potentiel de production très important. En RDC, la culture du riz, et en particulier le riz pluvial, revêt d'une importance capitale. Cette production essentiellement paysanne, ne couvre pas les besoins sans cesse croissants de la demande. Cependant, l'Institut National 
pour l'Etude et la Recherche Agronomiques (INERA), par l'entremise de son Programme National de Recherche sur le Riz (PNRR) a entrepris depuis 1987 la promotion de la culture du riz par l'amélioration aussi bien du rendement que de la qualité des variétés cultivées (Tollens et Biloso, 2006).

En 1992, la RDC a consommé 500.000 tonnes de riz dont 150.000 tonnes provenant de l'importation. Les cultigènes de plus en plus utilisés par les agriculteurs, victimes de contraintes biotiques et abiotiques sont actuellement menacés d'érosion génétique (Kasongo et al., 2003). C'est ainsi que le Centre de Riz pour l'Afrique ADRAO a, au cours de ces dernières années, mis un accent particulier sur la recherche et le développement participatif afin de réadapter les systèmes de production de riz aux nouvelles technologies plus appropriées et de faire face aux contraintes prioritaires (Sido et al., 2006).

Il est bien établi qu'environ $23 \%$ de toutes les calories consommées dans le monde proviendraient du riz. En outre, le riz a la plus forte digestibilité des protéines. Il est riche en énergie et constitue une bonne source de protéines. Un autre avantage du riz est sa faible teneur en fibres alimentaires et en tanins. C'est l'une des principales sources d'énergie, de protéines, de fer, de calcium et de vitamines telles que la thiamine, riboflavine et en niacine (Tollens et Biloso, 2006).

Dans un contexte extrêmement difficile en termes de sous-alimentation et de malnutrition que connaît la RDC, il est donc indispensable que des nouvelles variétés de riz soient vulgarisées en vue d'apporter une réponse appropriée aux groupes vulnérables. Ceci permettrait de redonner de la valeur aux grands bassins rizicoles du pays. Le riz est aussi important dans la prévention et le traitement des maladies (Tollens et Biloso, 2006).

Il est scientifiquement démontré que la consommation du riz permet de traiter des maladies telles que le diabète et la diarrhée. En effet, une étude chez près de
150 diabétiques (types 1 et 2) a montré que la portion soluble $\mathrm{du}$ son de riz était particulièrement efficace pour atténuer l'hyperglycémie, amenant même le quart d'entre eux à diminuer leur dose quotidienne d'insuline ou d'hypoglycémiants oraux au cours de l'étude. En outre, l'eau provenant de la cuisson du riz est considérée comme utile pour aider à traiter les diarrhées légères à modérées, en réduisant notamment le nombre de selles et en améliorant leur consistance (Tollens et Biloso, 2006).

Par rapport aux valeurs énergétiques, le riz représente dans les pays en développement environ $27 \%$ de l'apport énergétique et jusqu'à $60 \%$ pour plus de 2 milliards d'Asiatiques. Le riz est avant tout un fournisseur de glucides complexes (78\%), essentiellement sous forme d'amidon. Quant aux Apports vitaminique et minéral, le riz est une bonne source de vitamines B1 et B2, importantes pour l'équilibre et la bonne santé du système nerveux (ADRAO, 2003).

Les résultats obtenus dans cette étude montrent que toutes les lignées testées présentent un meilleur résultat par rapport au témoin local (SIPI). En effet, ces lignées sont plus productives, performantes et résistantes à la verse. $\mathrm{Eu}$ égard à toutes ces caractéristiques, ces lignées peuvent servir de source d'énergie et de vitamines pour nos populations pauvres.

A notre connaissance, c'est pour la première fois qu'une étude sur le riz NERICA de bas-fond est réalisée en RDC.

\section{Conclusion}

Les résultats obtenus ont montré que sur le plan de la susceptibilité, toutes les quatre lignées de riz NERICA présentent la résistance vis-à-vis de la pyriculariose. Au point de vue agronomique, il faut noter que toutes les lignées présentent une résistance assez bonne à la verse. Toutes ces lignées sont bonnes, présentant une bonne excertion paniculaire et portent beaucoup de grains par panicule avec un poids de 1000 grains élevé ainsi qu'un fort tallage supérieur au témoin local SIPI. Cependant, l'analyse de la teneur 
en amylose des grains et le test de cuisson devraient être préconisés en vue de déterminer la meilleure lignée parmi les quatre pouvant faire l'objet de la vulgarisation comme variété de culture répondant aux exigences des agriculteurs du point de vue qualités culinaires.

\section{REFERENCES}

ADRAO. 2003. Nerica en plein essor; un symbole d'espoir pour les rizicultures africaines. Abidjan.

Angladette A. 1966. Le Riz. Techniques Agricoles et Productions Tropicales. Maisonneuve et Larose: Paris.

Bouet A, Valets M, Keli ZJ. 2006. Une méthode d'évaluation de la résistance de variétés de riz vis-à-vis de la pyriculariose du cou au champ. Afria Rice Center (WARDA), 12(2): 193.

Cissoko M, Boisnard A, Rodenburg J, Press MC, Scholes JD. 2011. New Rice for Africa (NERICA) cultivars exhibit different levels of post-attachement resistance against the parasitic weeds Striga hermontica and Striga Asiatica. New Phytol., 192(4): 952-963.

Courtois B, Taillebois JE. 1990. Comportement en androgénèse de lignées de riz porteuses d'une stérilité mâle d'origine génocytoplasmique. Agronomie Tropicale, 45(2): 101-105.

Halidou A, Sido AY, Toudou A. 2006. Evaluation de lignées de riz pour leur compétitivité vis-à-vis des adventices. Africa Rice Center (WARDA), 12(2): 157161.

IRRI. 1980. Standard Evaluation System for Rice. International Rice Testing Program ( $2^{\text {nd }}$ edn). IRRI: Manila, Los Baños.

Kabuyaya D. 2001. Le Protocole «PRERP». La mise en place d'un essai et la collecte des données. Coopération Agricole Italienne à la RDC, At.C/PNR, Kinshasa.

Kasongo KM, Walangululu MJ, Bantodia KM, Likoko B, Mbuya K. 2003. Etude du comportement et des performances de huit lignées hybrides de riz pluvial à cycle moyen sélectionnées à Yangambi. Tropicultura, 21(3): 112 - 116.

Kone B, Etiene JB, Amadji G, Diata S. 2008. Caractérisation de la tolerance de NERICA à la secheresse de mi-saison en riziculture pluviale. African Crop Science Journal, 16(2): 133-145.

Koudjega T, Aboa K, Tossah BK, Tsatsu KD. 2006. Détermination des doses optimales d'azote pour l'amélioration des rendements de cinq variétés de riz de bas fond. Afria Rice Center (WARDA), 12(2): 17-23.

Narteh LT, Tenywa JS, Nampala P, Kawube G. 2006. Africa Rice Congress; Beyond the First Generation NERICAS in Africa: paradigms and partnerships for the next decade. Dar es Salaam, Tanzania.

Nseye MF, Aliou D, N'cho S, Dibue D. 2009. Situation de la riziculture en République Démocratique du Congo; Etude de Référence, PNR/INERA. Kinshasa.

PNR. 1993. Riz pluvial. Programme National Riz, Projet PNUD/FAO/ZAI/92/001. Kinshasa.

Sido YA, Basso A, Issaka S, Halidou A, Abdou S, Ibroh G. 2006. Evaluation des lignées intra et interspécifiques de riz de bas fond/irrigué au Niger. Africa Rice Center (WARDA), 12(2): 9-12.

Tollens E, Biloso A. 2006. République Démocratique du Congo: Profil des marchés pour les évaluations d'urgence de la sécurité alimentaire, Katholieke Universiteit Leuven, PAM, ODAN, Kinshasa.

Tshibambe PL. 2006. Introduction des variétés et lignées prometteuses par le Programme National Riz: impact sur l'augmentation de la production du riz en RD Congo. Afria Rice Center (WARDA), 12(2): 35-37.

Yovo K. 2008. Incitation par les prix, rentabilité et compétitivité de la production du riz au sud Togo, Rapport de recherche, Lomé. 